\title{
Dual benefit of bile acid receptor agonist
}

Bile acids, which contribute to the emulsification and digestion of lipid-soluble nutrients following a meal, are increasingly appreciated as also having important roles in metabolic signalling pathways. Writing in Cell Metabolism, Thomas and colleagues identify an agonist of $\mathrm{G}$ protein-coupled bile acid receptor 1 (GPBAR1; also known as TGR5) that is capable of both preventing weight gain and maintaining glucose homeostasis, revealing a possible new treatment for diabetes and obesity.

Activation of GPBAR1 by bile acid has previously been shown to increase energy expenditure in muscle and brown fat, preventing or reversing diet-induced obesity in mice. The recent identification of GPBAR1 expression in enteroendocrine L cells has led to speculation that GPBAR1 might also modulate glucose tolerance, as these cells secrete the incretin glucagon-like peptide 1 (GLP1), which increases insulin secretion in response to elevated blood glucose levels. To investigate this, Thomas and colleagues developed a potent and selective semisynthetic GPBAR1 agonist - a-ethyl-23(S)-methyl-cholic acid (referred to as INT-777) — based on the human bile acid cholic acid.

Confirming the known effects of GPBAR1 activation on energy expenditure, INT-777 increased mitochondrial activity, cellular oxygen consumption and ATP production in cell lines and in mice kept on a high-fat diet. As a result, the typical diet-induced increase in body weight was significantly attenuated in treated mice and fat mass was reduced compared with controls, showing the potential of INT-777 as an anti-obesity agent. Liver function also improved, indicated by reduced steatosis, fibrosis and serum markers of liver damage.

The authors next investigated whether GPBAR1 is involved in intestinal GLP1 release. INT-777 increased $\mathrm{Ca}^{2+}$ influx in a human enteroendocrine cell line, which was enhanced in the presence of glucose and correlated with a rise in GLP1 release. Moreover, on a high-fat diet, mice that overexpressed GPBAR1 showed improved glucose tolerance and increased GLP1 secretion and insulin release compared with control mice. The converse was seen in GPBAR1-knockout mice. Similarly, INT-777 increased GLP1 secretion in response to glucose in control mice, but this effect was blunted in mice lacking GPBAR1. Finally, to assess the therapeutic potential of INT-777 in type 2 diabetes, insulin-resistant diet-induced obese mice and diabetic $(\mathrm{db}-\mathrm{db})$ mice were treated with the compound for 8 weeks. Glucose homeostasis and insulin responses were normalized in treated mice

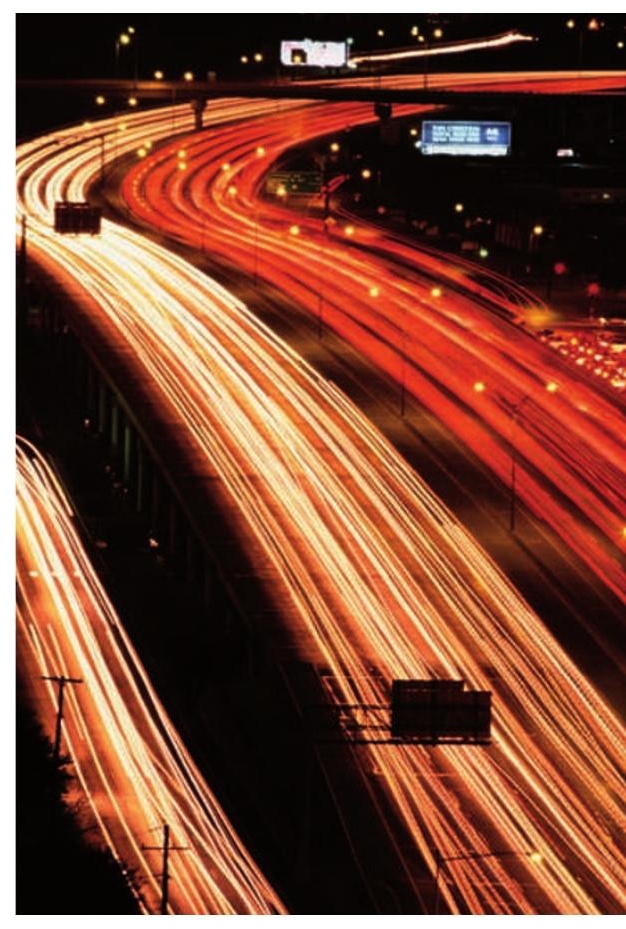

compared with controls. These effects were due to improved pancreatic function and reduced insulin resistance in the liver and muscle, and were not observed in GPBAR1-knockout mice.

Given the range of beneficial metabolic effects mediated by GPBAR1 activation, agonists of this receptor could represent a new class of agents for the management of diabetes, obesity and associated metabolic disorders. INT-777 is anticipated to enter clinical trials in 2010.

Sarah Crunkhorn

ORIGINAL RESEARCH PAPER Thomas, C. et al. TGR5-mediated bile acid sensing controls glucose homeostasis. Cell Metab. 10, 167-177 (2009) FURTHER READING Thomas, C. et al. Targeting bile-acid signalling for metabolic diseases. Nature Rev. Drug Discov. 7, 678-693 (2008) 